\title{
CASE FOR DIAGNOSIS
}

\section{A turbulent cause of bilateral pneumonia}

\section{E. Bathoorn*, M.A. Heitbrink" ${ }^{\#}$ C.L.A. Reichert ${ }^{\star}$ and W.G. Boersma*}

\section{CASE HISTORY}

A 62-yr-old female presented with complaints of progressive chest pain, increasing in severity with inspiration, a productive cough and fever for 2 days. Moreover, she had lost $12 \mathrm{~kg}$ in weight in the last 3 months and was experiencing night sweats. During this period, she had a folliculitis in her axilla. Her medical history yielded diabetes mellitus type II, hypertension and anaemia. Transthoracic echocardiography, performed 4 years ago, had shown flow abnormalities in the pulmonary artery and left ventricular volume overload. The patients' medication included metformin, insulin, hydrochlorothiazide, simvastatin, metoprolol, ferrofumerate and vitamin B.

\section{TABLE 1 Summary of investigations}

\begin{tabular}{|c|c|}
\hline Investigations & Result \\
\hline Leukocytes $\times 10^{9} \cdot \mathrm{L}^{-1}$ & 11.5 \\
\hline Neutrophils \% & 81.5 \\
\hline C-reactive protein $\mathrm{mg} \cdot \mathrm{L}^{-1}$ & 174 \\
\hline Erythrocyte sedation rate $\mathrm{mm} \cdot \mathrm{L}^{-1} \mathrm{~h}$ & 103 \\
\hline Urea nitrogen & Within normal ranges ${ }^{\#}$ \\
\hline Creatinine & Within normal ranges ${ }^{\#}$ \\
\hline Liver enzymes & Within normal ranges ${ }^{\#}$ \\
\hline Glucose $\mathrm{mmol} \cdot \mathrm{L}^{-1}$ & 9.0 \\
\hline D-dimer $\mu \mathrm{g} \cdot \mathrm{L}^{-1}$ & 1819 \\
\hline Haemoglobin $\mathrm{mmol} \cdot \mathrm{L}^{-1}$ & 5.5 (normocytic) \\
\hline Anti-nucleolar antibodies & $1: 40$ \\
\hline \multicolumn{2}{|l|}{ Arterial blood gases } \\
\hline $\mathrm{pH}$ & 7.51 \\
\hline $\mathrm{Pa}, \mathrm{CO}_{2} \mathrm{mmHg}$ & 34 \\
\hline $\mathrm{Pa}, \mathrm{CO}_{2} \mathrm{mmHg}$ & 68 \\
\hline Standardised $\mathrm{HCO}_{3} \mathrm{mmol} \cdot \mathrm{L}^{-1}$ & 28.4 \\
\hline Base excess & 4.5 \\
\hline Oxygen saturation \% & 96 \\
\hline Blood cultures & Negative \\
\hline Sputum cultures & Negative \\
\hline \multicolumn{2}{|c|}{$\begin{array}{l}\mathrm{Pa}_{1} \mathrm{CO}_{2} \text { : arterial carbon dioxide tension; } \mathrm{Pa}, \mathrm{O}_{2} \text { : arterial oxygen tension. }{ }^{\#} \text { : norma } \\
\text { ranges of: serum urea } 3-7 \mathrm{mmol} \cdot \mathrm{L}^{-1} ; \text { creatinine } 53-97 \mu \mathrm{mol} \cdot \mathrm{L}^{-1} \text {; aspartat } \\
\text { aminotransferase }<30 \text { units } \cdot \mathrm{L}^{-1} \text {; alanine aminotransferase }<35 \text { units } \cdot \mathrm{L}^{-1} ; \text { and } \\
\text { glutamyl transpeptidase }<35 \text { units } \cdot \mathrm{L}^{-1} \text {. }\end{array}$} \\
\hline
\end{tabular}

On physical examination, a pale and cachectic patient was seen. Her body temperature was $39^{\circ} \mathrm{C}$, with a heart rate of 104 beats $\cdot \mathrm{min}^{-1}$, respiratory rate of 20 breaths $\cdot \mathrm{min}^{-1}$ and blood pressure of $160 / 65 \mathrm{mmHg}$. Chest auscultation revealed normal breath sounds, a $2 / 6$ systolic murmur in the right parasternal area and a $1 / 6$ continuous murmur in the left parasternal area. Otherwise, physical examination was normal.

An overview of investigations is presented in table 1. A chest radiograph was performed upon admission (fig. 1) and a contrast-enhanced computed tomography (CT) scan was performed before and after treatment (figs 2 and 3, respectively). A CT scan was also obtained in the parenchymal setting (fig. 4).

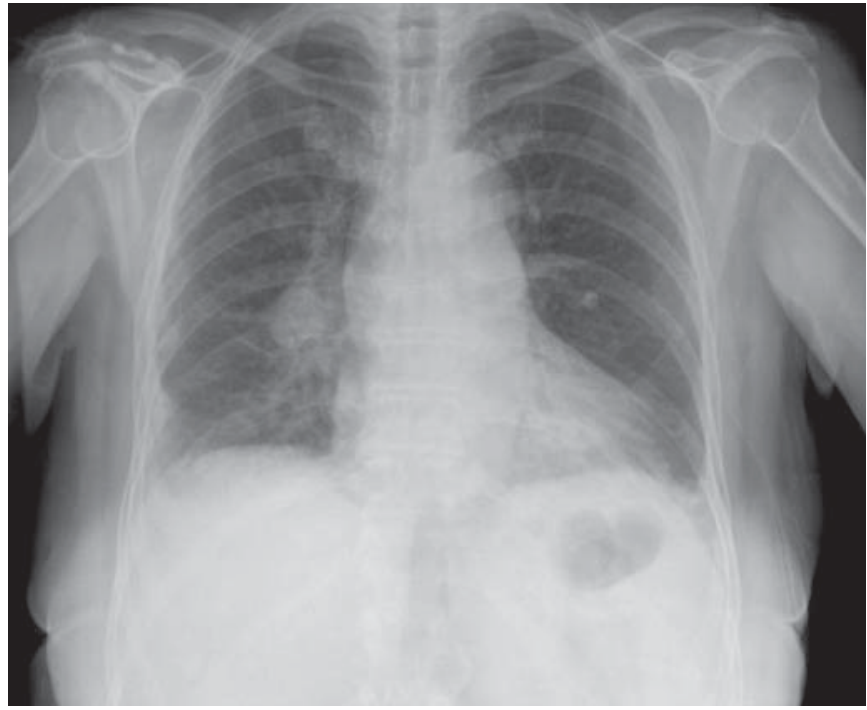

FIGURE 1. Chest radiograph on admission showing a large right hilum and consolidations in both lower lobes.

Depts of *Pulmonology, ${ }^{*}$ Radiology, and ${ }^{*}$ Cardiology, Medical Center Alkmaar, Alkmaar, The Netherlands. STATEMENT OF INTEREST: None declared. 


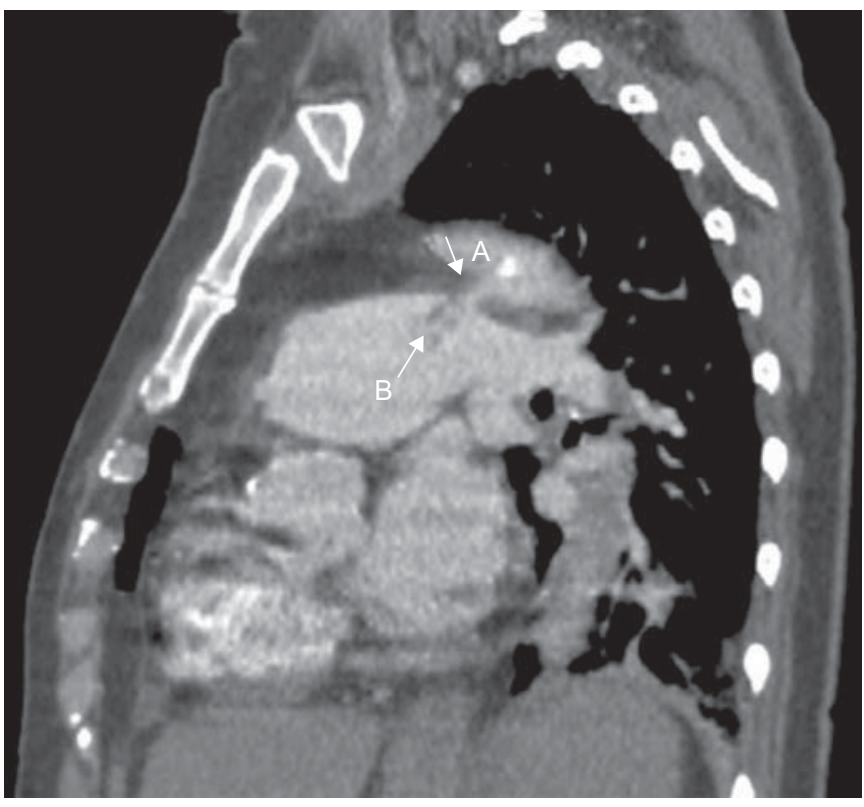

FIGURE 2. Contrast-enhanced computed tomography images at admission. The sagittal plane is shown, which has been reconstructed from data of transverse slices. Arrow A indicates the patent ductus arteriosus connecting the aortic arch to the pulmonary artery. Arrow $B$ indicates opacities in the pulmonary trunk suggestive of vegetations. A calcified area is visible in the aortic arch.

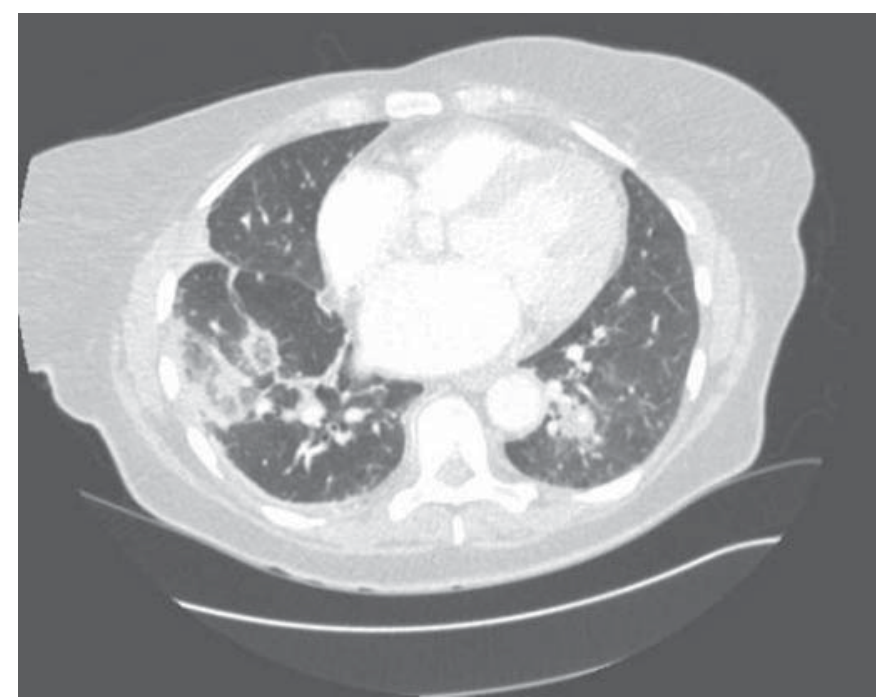

FIGURE 4. Computed tomography of the lung basis. There are bilateral consolidations in the lower lobes.

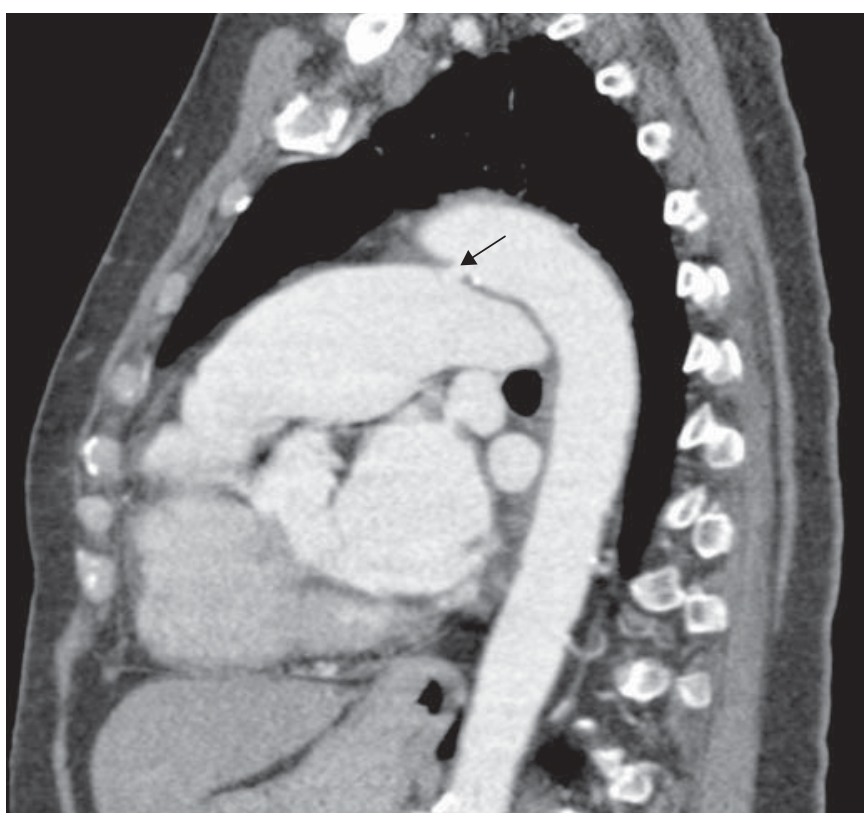

FIGURE 3. Contrast-enhanced computed tomography images 4 months after antibiotic treatment. The sagittal plane is shown, which has been reconstructed from data of transverse slices. The arrow indicates the patent ductus arteriosus, connecting the aortic arch to the pulmonary artery. The vegetations in the pulmonary trunk have disappeared.

BEFORE TURNING THE PAGE, INTERPRET THE PATIENT HISTORY, CHEST RADIOGRAPHS, CONTRAST-ENHANCED CT SCANS AND THE CT SCAN, AND SUGGEST A DIAGNOSIS. 


\section{INTERPRETATION}

The patient had a history of fatigue, fever, weight loss and anaemia, which might have been caused by chronic infection or malignancy. The chest radiograph on admission showed a large right hilum and consolidations in both lower lobes (fig. 1). CT scanning confirmed a patent ductus arteriosus (PDA), which had been suspected on transthoracic echocardiography 4 yrs earlier, and enlargement of both right and left pulmonary arteries. Furthermore, it showed opacities in the pulmonary trunk suggestive for vegetations. A calcified area was found in the aortic arch, which might indicate previous infection (fig. 2). No signs of embolisms in the pulmonary arteries were seen. In the parenchymal setting, bilateral consolidations in the lower lobes were present (fig. 4). Transoesophageal colour Doppler echocardiography showed a hyperdynamic left ventricle, enlarged left atrium, a turbulent flow in the pulmonary artery and an abnormal flow from the aorta to the pulmonary artery through a PDA.

\section{Diagnosis: Pulmonary endarteritis in the presence of a PDA and subsequent metastatic pulmonary infection.}

In the patient, vegetations were shown by CT scans in the pulmonary trunk downstream of PDA. In this area, the endothelium is damaged by the turbulent jet. The damaged endothelium is very susceptible for infections with growth of vegetations, particularly in the case of recurrent bacteraemia from an infective source. These vegetations might have formed septic emboli, causing a sudden deterioration of symptoms and pulmonary consolidations. Therefore, the imaging tests, combined with the clinical picture, resulted in the diagnosis of pulmonary endarteritis in the presence of a PDA and subsequent metastatic pulmonary infection.

\section{TREATMENT AND CLINICAL COURSE}

The patient was initially treated with amoxicilline-clavulanate $750 \mathrm{mg}$ t.i.d. during the first 6 days. This was changed to penicillin G $12 \times 10^{6} \mathrm{IU} \cdot$ day $^{-1}$ i.v. continuously, and gentamicin $260 \mathrm{mg}$ q.d. i.v. for 4 weeks when the diagnosis of pulmonary endarteritis was considered most likely. Numerous blood cultures were negative. Furthermore, she was treated with low molecular weight heparin and coumarin. This treatment resulted in normalisation of temperature and C-reactive protein. However, after 2 weeks of treatment, the patient again developed cough and fever. A bronchoalveolar lavage specimen grew $\beta$-lactamase producing Moraxella catarrhalis resistant to penicillin. Therefore, antibiotic treatment was extended with cefuroxime i.v. for 1 week. A chest radiograph showed a new opacity in the left upper field and positron emission tomography-CT showed increased activity in this area. The opacity in the pulmonary artery had disappeared. After 4 weeks, the patient was discharged without any signs of chest symptoms or fever. During follow-up, the patient complained of fatigue, without recurrence of infection, and CT scans showed the PDA without opacities in its flow (fig. 3). The patient underwent percutaneous closure of the ductus arteriosus using Amplatzer ductal closure device (AGA Medical Corporation, Golden Valley, MN, USA) 8 months later. Successful closure of the PDA was confirmed by colour Doppler echocardiography, showing no residual shunt from the aortic arch to the pulmonary trunk. After closure, the patient was free of complaints.

\section{DISCUSSION}

The presented case showed endovascular complications of PDA in a 62-yr-old-patient. The ductus arteriosus is a shunt connecting the aortic arch to the pulmonary artery. It is part of the foetal vascular system, allowing blood to bypass the fluid filled lungs. Normally, the ductus closes when the newborn child starts breathing, and it is completely sealed within the first 10 days after birth. However, it is not uncommon that the ductus does not completely close. This vascular state, a PDA, is a risk factor for pulmonary endarteritis, embolism, pulmonary hypertension and cardiac failure.

In the presented case the PDA was diagnosed 4 yrs earlier with a ratio between pulmonary and systemic flow of $<1.5$. However, further workup to evaluate and close the ductus was refused by the patient in the absence of symptoms at the age of 60 yrs.

During the first half of the 21st Century, pulmonary endarteritis was a common cause of death in patients with a PDA [1]. Due to the introduction of antibiotics, and the development of surgical techniques to close the PDA, the risk of endarteritis and mortality has been substantially decreased, and is nowadays very rare [2]. A recent study in Sweden reviewed the records of 281 patients with PDA representing an aggregate of 1,196 yrs at risk of infective endarteritis. The study did not find any case of endarteritis [3]. This raised the question, whether closure of a PDA is indicated for the sole purpose of prevention of infective endarteritis [3,4].

A few cases of a PDA accompanied by pulmonary endarteritis, with or without subsequent pulmonary infiltrations, have been reported [5-11]. Blood cultures in these case reports grew Gamella species, group D Streptococcus, Streptococcus mitis, S. capitis, S. millei, and $S$. viridans. In our case, no species grew in the numerous blood cultures, probably because the patient had been treated with oral antibiotics by her general practitioner. The patient was treated with antibiotics for 4 weeks, as is recommended for endocarditis lenta with negative cultures, with i.v. penicillin and gentamicin [12]. In a specimen obtained by brochoalveolar lavage, Moraxella catharralis was isolated. This pathogen was probably not the cause of endarteritis of PDA as it rarely grows invasively.

Endocarditis in general is almost always caused by recurrent bacteraemia from an infective source, and spontaneously in $<1 \%$ [13]. In our case, we can only speculate on the source of infection. The start of the patients' chronic complaints was accompanied by folliculitis in her armpit, for which she might have been more susceptible due to her diabetes. Whether diabetes is an extra risk factor for infective endarteritis is unsure. However, a case with the combination diabetes, PDA, and infective pulmonary endarteritis with subsequent pulmonary infiltrates has been described previously [5]

The differential diagnoses included not only infective endarteritis and subsequent embolism, but also embolisms due to a noninfectious inflammatory process in the flow area of the PDA, a malignant tumour or an autoimmune disease.

The diagnosis of infectious endarteritis is difficult when blood cultures are negative. Not all endocarditis pathogens grow in blood, for example, Bartonella spp, Coxiella spp, Chlamydia spp 
and HACEK-group species are fastidious [14, 15]. In the case of negative blood cultures, inflammatory markers may differentiate between infectious and noninfectious causes of disease. To establish the diagnosis infectious endarteritis, C-reactive protein, procalcitonin, interleukin- 6 , tumour necrosis factor- $\alpha$ and interleukin-1 $\beta$ in serum could be useful, since these markers are significantly increased compared with healthy control subjects. Of these markers, C-reactive protein has the best predictive value [16]. Increased C-reactive protein, combined with fever, appears to indicate in the direction of an infectious cause. With this information, we consider noninfectious embolisms less likely.

The rapid disappearance of the opacity after starting the treatment advocates against a malignant tumour. The patient had positive titres of anti-nucleolar antibodies, which could indicate an autoimmune cause of her complaints. However, the patient did not receive any immune modulating treatment, and responded to antibiotics with a complete normalisation of Creactive protein and body temperature.

The clinical course, blood examination and response to therapy, together with the results of the imaging tests, nearly proved the diagnosis infective endarteritis and subsequent pulmonary embolisms, although we did not culture a pathogenic microorganism. However, a positive blood culture is not a necessity for the diagnosis pulmonary endarteritis as based on the definition used in a retrospective study, in which PDA accompanied with pulmonary endarteritis is defined as the clinical picture typically associated with echocardiographic vegetations, or positive blood cultures [3].

In conclusion, pulmonary endarteritis is a rare, life-threatening complication of a patent ductus arteriosus. In the case of unexplained fever in a patient with a pre-existing patent ductus arteriosus, pulmonary endarteritis should be considered and investigated by echocardiography, computed tomography angiogram or magnetic resonance imaging and blood cultures. As a consequence of septic emboli, pulmonary infiltrates may develop.

\section{REFERENCES}

1 Campbell M. Natural history of persistent ductus arteriosus. Br Heart J 1968; 30: 4-13.

2 Awadallah SM, Kavey RE, Byrum CJ, et al. The changing pattern of infective endocarditis in childhood. Am J Cardiol 1991; 68: 90-94.
3 Thilen U, Astrom-Olsson K. Does the risk of infective endarteritis justify routine patent ductus arteriosus closure? Eur Heart J 1997; 18: 503-506.

4 Sullivan ID. Patent arterial duct: when should it be closed? Arch Dis Child 1998; 78: 285-287.

5 Onji K, Matsuura W. Pulmonary endarteritis and subsequent pulmonary embolism associated with clinically silent patent ductus arteriosus. Intern Med 2007; 46: 1663-1667.

6 Rivera IR, Moises VA, Brandao AC, et al. Patent ductus arteriosus and pulmonary artery endarteritis. Arq Bras Cardiol 1997; 69: 335-338.

7 Bilge M, Uner A, Ozeren A, et al. Pulmonary endarteritis and subsequent embolization to the lung as a complication of a patent ductus arteriosus-a case report. Angiology 2004; 55: 99-102.

8 Vargas-Barron J, Attie F, Buendia-Hernandez A, et al. Echocardiographic recognition of pulmonary artery endarteritis in patent ductus arteriosus. Am Heart J 1985; 109: 368-370.

9 Lankipalli RS, Lax K, Keane MG, et al. Images in cardiovascular medicine. Infected patent ductus arteriosus. Circulation 2005; 112: e364-e365.

10 Celebi A, Erdem A, Cokugras Het al, Infective endarteritis in a 2-month-old infant associated with silent patent ductus arteriosus. Anadolu Kardiyol Derg 2007; 7: 325-327.

11 Yanyk A, Yetkin E, Lleri M, et al. Vegetation due to Streptococcus viridans in the pulmonary artery in a child with patent ductus arteriosus. Int J Cardiol 2000; 72: 189-191.

12 Elliott TS, Foweraker J, Gould FK, et al. Guidelines for the antibiotic treatment of endocarditis in adults: report of the Working Party of the British Society for Antimicrobial Chemotherapy. J Antimicrob Chemother 2004; 54: 971-981.

13 Durack DT. Prevention of infective endocarditis. N Engl J Med 1995; 332: 38-44.

14 Houpikian P, Raoult D. Blood culture-negative endocarditis in a reference center: etiologic diagnosis of 348 cases. Medicine (Baltimore) 2005; 84: 162-173.

15 Das M, Badley AD, Cockerill FR, et al. Infective endocarditis caused by HACEK microorganisms. Annu Rev Med 1997; 48: 25-33.

16 Watkin RW, Harper LV, Vernallis AB, et al. Pro-inflammatory cytokines IL6, TNF- $\alpha$, IL1 $\beta$, procalcitonin, lipopolysaccharide binding protein and C-reactive protein in infective endocarditis. J Infect 2007; 55: 220-225. 